\title{
Cardiolipin IgG Antibody Measurement
}

National Cancer Institute

\section{Source}

National Cancer Institute. Cardiolipin Ig G Antibody Measurement. NCI Thesaurus. Code C111144.

The determination of the amount of cardiolipin Ig G antibody present in a sample. 